Isotropic-nematic transition and cholesteric phases of helical Yukawa rods

A. Kuhnhold and T. Schilling

Citation: J. Chem. Phys. 145, 194904 (2016); doi: 10.1063/1.4967718

View online: http://dx.doi.org/10.1063/1.4967718

View Table of Contents: http://aip.scitation.org/toc/jcp/145/19

Published by the American Institute of Physics 


\title{
Isotropic-nematic transition and cholesteric phases of helical Yukawa rods
}

\author{
A. Kuhnhold ${ }^{\text {a) }}$ and T. Schilling \\ Theory of Soft Condensed Matter, Physics and Materials Science Research Unit, Université du Luxembourg, \\ L-1511 Luxembourg, Luxembourg
}

(Received 27 July 2016; accepted 1 November 2016; published online 17 November 2016)

\begin{abstract}
We present a Monte Carlo simulation study of helical Yukawa rods as a model for chiral liquid crystal mesogens. To simulate the cholesteric phase, we introduce a new simulation method that uses soft walls and self-determined boundary conditions. We observe that the isotropic-nematic phase transition is shifted to lower volume fractions with decreasing salt concentration as well as with increasing internal pitch of the rods. For particular sets of interaction parameters, the sense of the cholesteric pitch inverts, i.e., depending on concentration, mesogens of a given handedness can produce cholesteric phases of both chiral senses. Published by AIP Publishing. [http://dx.doi.org/10.1063/1.4967718]
\end{abstract}

\section{INTRODUCTION}

Liquid crystals that consist of chiral mesogens show a rich phase behaviour. In addition to the orientationally disordered isotropic phase and the nematic and smectic phases, in which the mesogens are aligned along a director, they form cholesteric phases, in which the nematic director rotates around the cholesteric axis. ${ }^{1}$ The pitch of this rotation determines the optical properties of the liquid crystal, e.g., the wavelength of reflected or transmitted light. ${ }^{2,3}$ This property is the basis for numerous applications on the market as well as for ideas of future applications that involve chiral liquid crystals, as e.g., sensors which change colour if the pitch reflects a change of the chemical environment, the humidity, or the strain on a material. $^{4-6}$

Thermotropic chiral liquid crystals can be made from synthesized molecules. A few out of many examples are chiral biphenyls, ${ }^{7}$ bis-(binaphtylenedioxy)silane, ${ }^{5}$ ester derivatives of hydroxypropylcellulose, ${ }^{8} \mathrm{P} 6-\mathrm{M},{ }^{9}$ or copolymers consisting of $\gamma$-benzyl glutamate and $\gamma$-alkyl glutamate. ${ }^{10}$ But also natural chiral mesogens can be found, e.g., DNA, $f d$ virus, and cellulose nanocrystals (CNC) ${ }^{11-14}$ Currently, effort is put into research on CNC, since they are available in large amounts, renewable, and biodegradable. The aim is to eventually use $\mathrm{CNC}$ in technological applications, but first one needs to control the properties of the resulting liquid crystal structures. In this paper, we address this issue by means of Monte Carlo simulations of a model for CNC. We show results for parameters that are realistic for experimental studies on $\mathrm{CNC}$, but we do not restrict our work to these parameter values. In the literature, different models of chiral mesogens are described (with varying degrees of chemical detail), which show a cholesteric phase under certain conditions. Studies of these are based on density functional theories ${ }^{15-23}$ or simulations. ${ }^{24-30}$ The model we have used is a hard spherocylinder around which a set of discrete point charges are wrapped in a helical arrangement. This model is similar to the one in Ref. 17 and almost the same

a)Electronic mail: anja.kuhnhold@uni.lu as in Ref. 29, where the hard spherocylinder is made of fused spheres with a WCA potential, to make it applicable to molecular dynamics simulations. Our study is delimited from these studies by the range of parameters that we have addressed.

Although we aim to compare results to cellulose nanocrystal suspensions, the model is also relevant to other chiral colloids, e.g., chitin nanofibrils with their electrostatic charge coat, ${ }^{31,32}$ rod-like particles from phytosterols that have chiral distribution of surface charge originating in chiral distribution of phytosterol and water molecules, ${ }^{33}$ and $f d$ viruses, where a polyelectrolyte (single stranded DNA) is coated by proteins in a helicoidal way. ${ }^{12}$

To equilibrate a cholesteric phase in a finite simulation volume is not straightforward, since the pitch needs to be commensurate with the dimensions of the simulation box, if standard periodic boundaries are used. To tackle this problem, we introduce a new method combining soft walls and self-determined boundary conditions.

\section{MODEL AND SIMULATION}

Each chiral molecule is modeled as a hard spherocylinder of length $L$ and diameter $D$ (called rod from here on) that has a helical distribution of $n^{\mathrm{pc}}$ point charges on its surface. The unit of length is the diameter $D$. Fig. 1 sketches this model for different internal pitches $p^{\text {int }}$ (length of one full turn of the charge helix).

Each pair of point charges on different rods interacts by a Yukawa potential,

$$
U_{Y}(r)=k_{B} T Z^{2} \lambda_{B} \frac{\exp [-\kappa r]}{r},
$$

where $r$ is the distance between the point charges, $Z$ the strength of the charges, $\lambda_{B}$ the Bjerrum length, $\kappa$ the Debye screening constant, $k_{B}$ Boltzmann's constant, and $T$ the temperature. $\lambda_{B}$ and $\kappa$ are defined as

$$
\begin{aligned}
& \lambda_{B}=e^{2} /\left(4 \pi \epsilon_{0} \epsilon_{r} k_{B} T\right), \\
& \kappa=\sqrt{4 \pi \lambda_{B}\left(\tilde{Z} \rho+2 c_{S}\right)},
\end{aligned}
$$




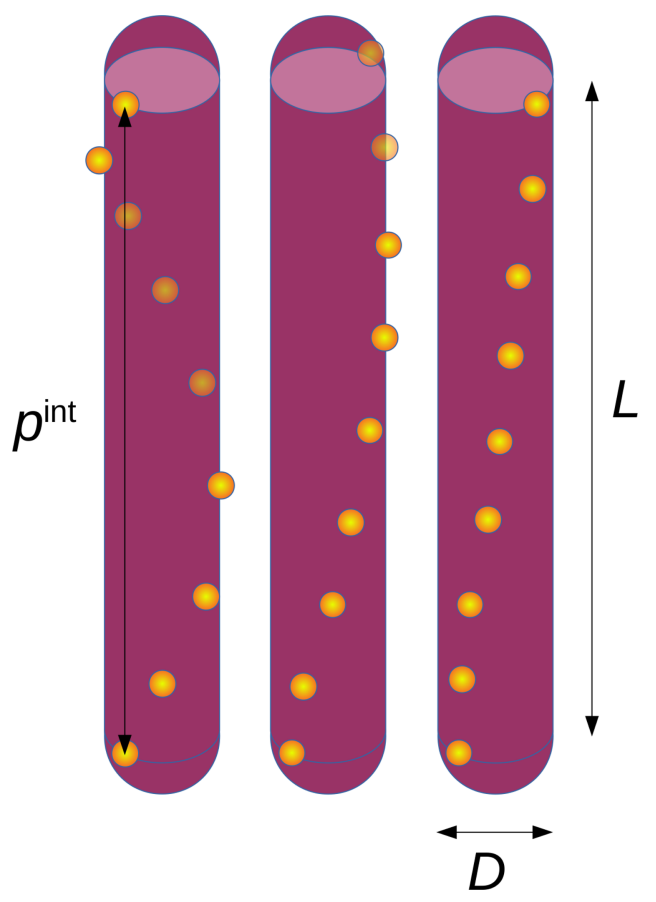

FIG. 1. Sketch of the simulation model with length $L$, diameter $D$, and different internal pitches (left $p^{\text {int }}=L$, middle $p^{\text {int }}=2 L$, right $p^{\text {int }}=4 L$ ), with the number of point charges $n^{\mathrm{pc}}=9$. Reprinted with permission from HonoratoRios et al., Front. Mater. 3, 21 (2016). Copyright 2016 Author(s), licensed under a Creative Commons Attribution 4.0 License.

with the number density of rods $\rho$ and the added salt concentration $c_{s} . \tilde{Z}$ is either the charge strength $Z$ or the total charge $Z^{s}=n^{\mathrm{pc}} Z$ (note: the terms total and surface charge are used interchangeably). Using the former, one can change $n^{\mathrm{pc}}$ without changing the interaction potential between the charges. In this way one can study the direct effect of changing the number of discrete point charges. However, the latter is more realistic, since the screening length depends on the number of counterions, which is proportional to the total charge on the rod, and therefore changes with the number of point charges.

We use Metropolis Monte Carlo in the NVT ensemble for all simulations. Periodic boundary conditions are either used in all three dimensions or restricted to two dimensions for the simulations of the cholesteric phase. Details of the treatment of the third dimension are described below. Starting configurations are prepared in the nematic phase and then released to form the different phases during equilibration runs. We apply single particle translations, axis rotations, and azimuthal rotations, where step sizes are adjusted to have acceptance rates of 0.5 . The minimum length of all production runs is $10^{5}$ Monte Carlo steps; usually $3 \cdot 10^{5}$ steps are analyzed to be sure that equilibration was sufficient and more steps are necessary for strongly fluctuating systems.

\section{BOUNDARIES FOR CHOLESTERIC PHASE}

In order to find equilibrium cholesteric pitches that do not match the box dimension, we treat the rods close to the boundaries in $z$-direction (the cholesteric axis) with a new method. The main idea of this method is to introduce soft walls that do not allow the rods to pass, but that do allow them to interact with periodic images of rods at the other end of the box.

In computer simulations, one often uses a small system with periodic boundaries to mimic a bulk system. This approach cannot be directly extended to the cholesteric phase. The most obvious strategy one might attempt to apply would be (1) to cut the box into thin slices perpendicular to the cholesteric axis (here the $z$-axis), (2) to rotate the slices with respect to each other, (3) to add periodic images to each slice in the (xy)-plane, and (4) to rotate the two outermost slices to match up when periodic images are needed in the $z$ direction. This straightforward strategy does not produce a simple continuation of the box into its periodic images. Interactions across the "corners" of the slices, where periodic images in the (xy)-plane interact along $z$, are not taken into account correctly.

In the literature, several attempts to solve the boundary problem by means of hard walls have been reported. ${ }^{26,30} \mathrm{In}$ these studies, standard periodic images were used in two directions, while in the third direction particles could neither cross the boundaries nor interact across them. Hard walls locally induce positional and orientational order, ${ }^{34-37}$ i.e., the order parameter in the nematic phase depends on the distance from the walls. (The nematic order parameter $S_{2}$ is defined as the largest eigenvalue of $Q_{i j}=\frac{1}{N} \sum_{\alpha=1}^{N} \frac{3}{2}\left(u_{\alpha}^{i} u_{\alpha}^{j}-\delta_{i j}\right)$, where $u_{\alpha}^{i}$ is the $\mathrm{i}$-th component of the unit vector along the axis of particle $\alpha$ and $N$ is the number of rods either in the total system or in a given subsystem as, e.g., a layer close to the wall. ${ }^{38}$ ) Since the cholesteric pitch depends on the nematic order parameter and on the density, one does not find the bulk equilibrium pitch close to a hard wall. Therefore, if hard walls are used, the box needs to be sufficiently large to allow for relaxation to the bulk behaviour in the center.

A method to calculate the equilibrium cholesteric pitch without using any walls is given in Ref. 25 . There the whole periodic image of the simulation box is rotated by an integer $\left(n_{p}\right.$, including 0 ) multiple of $\pi / 2$ (twisted periodic boundary conditions ${ }^{39}$ ), forcing the pitch to be $4 L_{z} / n_{p}$. Since this pitch is, in general, not the equilibrium one, one can measure the free energy or intermolecular torques for different values of $n_{p}$ and relate their difference, via quadratic or linear forms, respectively, to the equilibrium pitch, as described in Ref. 25. This method is used in Ref. 29, where a model that is very similar to ours is used.

Here we propose to use soft walls that allow a rotation of the rods into the wall and thus reduce the difference in orientational order across the simulation box. In this way, equilibrium pitches can be reached in much smaller boxes than in the case of hard walls. The degree of rotation of the rods into the walls is limited by a second, hard wall at a distance $\pm f \frac{L}{2}$ from the soft wall (see Fig. 2 left panel). The parameter $f$ sets the degree of softness; for $f=0$ the walls are hard with respect to rod positions and orientations, while for $f=1$ the rods are allowed to freely rotate into the wall, but not to move their centres past it.

The parameter $f$ is adapted iteratively in response to the difference between the average order parameter in the box $S_{\mathrm{av}}$ and the order parameter close to the walls $S_{\mathrm{w}}$,

$$
\tilde{f}\left(S(z), t_{i}\right)=\left(S_{w}-S_{a v}\right) / n,
$$



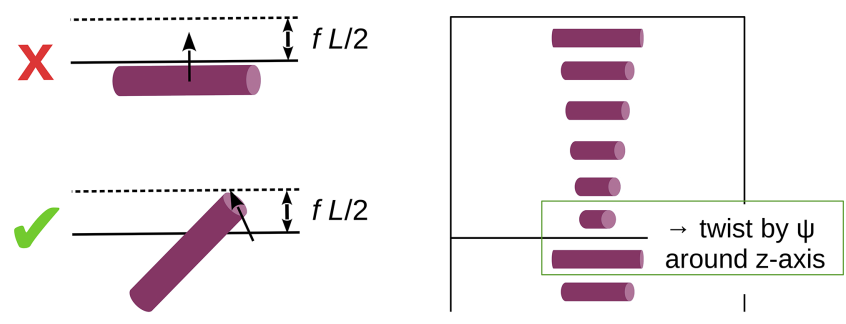

FIG. 2. Sketch of the new method: Center of mass cannot cross the wall; rotation into wall up to $f L / 2$ allowed; twist of periodic image by $\Psi$ for the interaction across the wall.

$$
\begin{aligned}
& f\left(t_{i}\right)=\sum_{k=0}^{i} \tilde{f}\left(S(z), t_{k}\right), \\
& \tilde{f}\left(t_{0}\right)=0,
\end{aligned}
$$

where $S_{\text {av }}$ is averaged over the order parameter $S(z)$ in slices that are stacked in $z$-direction, $n$ is the number of times the softness is adapted, and $t_{i}$ is the current simulation step. ${ }^{48}$ We use a $z$-dependent order parameter (the order parameter calculated for slices in $z$-direction) instead of the bulk order parameter, because the bulk value in an infinite cholesteric phase is 0.25 while it is 1 in the nematic phase as well as in a thin, perfectly nematic slice cut from a cholesteric phase. Thus to compare the order in the box to the order in a slice close to the wall, $S_{\mathrm{av}}$, needs to be averaged over slices rather than over all rods in the system at once. $^{49}$

The second ingredient of the new method is the interaction across the soft walls. The reader might ask, why at all one should calculate cross-boundary interactions in this confined system? The answer lies in the necessity of having a repulsive wall potential: If there was no (or even an attractive) wall potential, the rods close to the wall would behave very different from rods in bulk. They would minimize their energy by rotating the majority of the surface charges away from the center of the box. Instead of choosing an analytical repulsive potential, as used, e.g., in Ref. 40 , we want to mimic the bulk by using the repulsive interaction given by the system itself. This is done by calculating cross-boundary interactions. If we used the simple periodic image of a rod to calculate the interaction with another rod behind the wall, we would stabilize either the nematic phase or cholesteric phases with pitches $P=2 L_{z} / n_{p}$, where $n_{p}$ is an integer ( $n_{p}=0$ is the nematic phase). To be able to find pitches independent of the box length, the rods' periodic images are rotated by an angle $\Psi$, giving a pitch of $2 \pi L_{z} / \Psi$, and the equilibrium value of $\Psi$ is found via an additional Monte Carlo move that attempts to change $\Psi$ during the simulation. With this we stabilize the cholesteric phase by implying a continuous rotation. This method of rotating periodic images, known as self-determined boundary conditions, ${ }^{41,42}$ was used before only for lattice models. In systems with a repulsive (hard or soft) contribution, sufficiently large lattice spacing is needed, to be able to rotate all periodic images at once without creating overlaps, and so to be able to draw the periodic image of the whole simulation box. In addition only nearest neighbor interactions were considered and the interaction only depended on the relative orientation of the particles. In the continuum model, we only rotate one rod at a time to avoid overlaps. (This is possible since the potential is a pair potential.) Thus we cannot draw an image of the whole simulation box. The system is not periodic in the common sense-but each particle "sees" a well defined rotated periodic image of the box, which is used to calculate the particle's energy. In practice, this means whenever one needs to calculate a particle $k$ 's energy (after every single particle move and after the additional MC move that changes $\Psi$ ), the interaction with particles in the original box is calculated as usual and the interaction with periodic images is calculated with $k$ being rotated by $\Psi$ (but at fixed position). For the additional move to change $\Psi$, which is attempted after $N$ single particle moves on average, with a step size $\delta$ adjusted to have an acceptance rate of 0.5 , each particle's energy needs to be recalculated in this way with the new $\Psi$ to find the new system energy. The acceptance criterion is the usual Metropolis expression using the calculated new and old system energy.

Note that the interactions between periodic images in the $z$-direction are now not symmetric under exchange of the particles anymore, which was the case in lattice models with nearest neighbor interaction, where the positions are fixed, i.e., the potential between the particle $i$ and the periodic image of particle $j$ is not the same as the potential between the particle $j$ and the periodic image of particle $i$. Thus the Hamiltonian of a finite system with boundary conditions is not the same as of the bulk system. Clearly, this kind of interaction is unphysical. However, the effects on observables scale as the ratio of volume of a boundary layer to the system volume, as we show in the Appendix.

The soft wall method is an improvement over the hard wall method, but still the presence of walls and the finite system size introduce boundary effects, which are discussed in the Appendix.

\section{RESULTS}

\section{A. Isotropic-nematic transition}

In order to obtain a rough overview over the phase diagram, we first study the nematic order parameter $S_{2}$ in dependence on the volume fraction of rods, $\eta=N \pi$ $\left(L D^{2} / 4+D^{3} / 6\right) / V$, the internal pitch, the aspect ratio, the surface charge, and the salt concentration for systems with the usual periodic boundary conditions. As a consequence of these boundaries, the system is restricted to form phases without twist, which may not be the equilibrium phases. If we assume the cholesteric pitch to be much larger than the box length, the nematic phase, however, is a good approximation of the cholesteric phase.

Fig. 3 shows the nematic order parameter at the isotropicnematic (IN) transition of aspect ratio $L / D=10$ rods for different values of the internal pitch $p^{\text {int }}$ between $L / 2$ and $\infty$; multiple data points at the same concentration are results from independent runs. For decreasing internal pitch, the IN transition shifts to higher volume fraction, because the stronger 


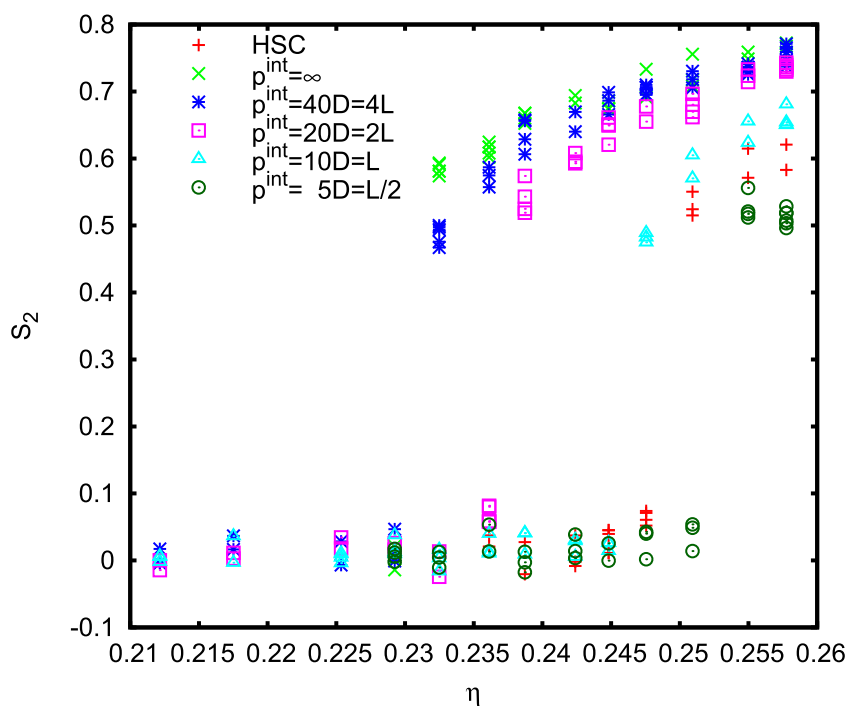

FIG. 3. Nematic order parameter vs. volume fraction for varying internal pitches $p^{\text {int }}$. $\left(N=1800, L=10 D, n^{\mathrm{pc}}=9, Z=10, c_{s}=1 D^{-3}\right)$. Also shown is the hard spherocylinder case (HSC).

twist of the charge helix induces a rotation of neighbouring rods against each other and thus reduces the local orientational order. The limiting case of infinite internal pitch stabilizes the nematic phase (as compared to the hard spherocylinder case), since the charges lie on a straight line and parallel alignment is favored. The screening length only depends on the volume fraction and ranges from $\kappa^{-1}=0.585 D$ to $0.594 D$. The effective shape of the rod is therefore that of a hard spherocylinder with an overlapping soft spherocylinder of diameter $1.18 D$ whose axis lies on the surface of the hard cylinder. The other limiting case is a vanishing internal pitch, which would result in a soft spherocylinder with diameter $2.18 \mathrm{D}$ and the hard spherocylinder as its core. This would give a decreased effective aspect ratio and therefore shift the IN transition to higher volume fractions compared to the hard spherocylinder case. The difference between an

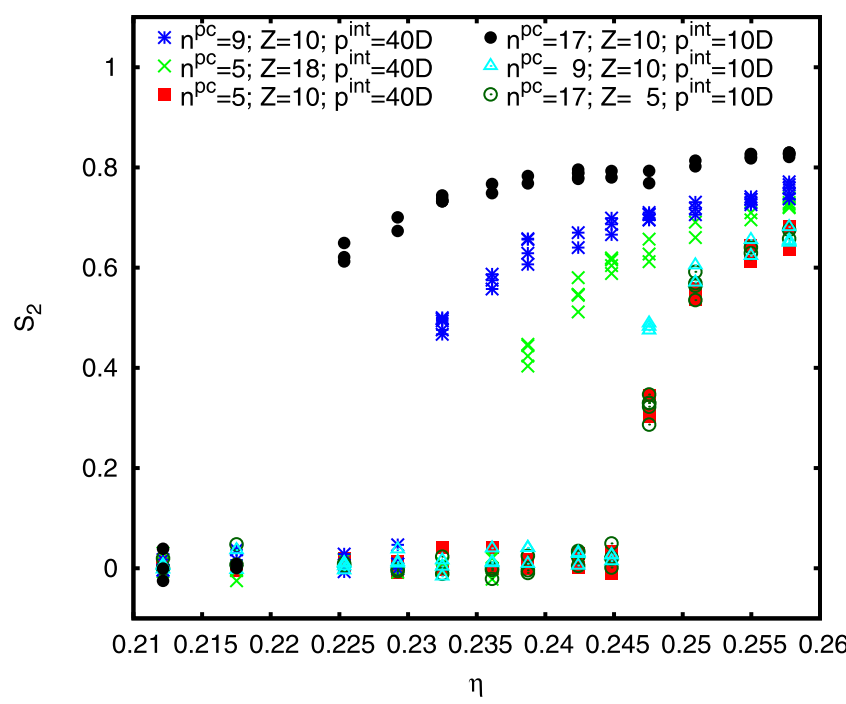

FIG. 4. Nematic order parameter vs. volume fraction for varying numbers of point charges $n^{\mathrm{pc}}$ and strength $Z\left(N=1800, L=10 D, c_{s}=1 D^{-3}\right)$. infinite internal pitch and an internal pitch of $4 L$ is almost negligible.

With increasing total charge $\left(n^{\mathrm{pc}} Z\right)$ from 50 to 90 , the nematic order increases and the IN transition shifts to a lower volume fraction (see Fig. 4, $p^{\text {int }}=40 D$ ). This shift also depends on the distribution of the total charge on the point charges: With 5 point charges of $Z=18\left(\kappa^{-1}=0.56 D\right)$, the nematic order is less than with 9 point charges of $Z=10\left(\kappa^{-1}=0.59 D\right)$. This difference becomes smaller with increasing number of point charges, as can be seen in Fig. $4\left(p^{\text {int }}=10 D\right)$ where the total charge decreases from 170 to 90 and 85 with decreasing nematic order, while the results for 17 point charges holding $Z=5\left(\kappa^{-1}=0.61 D\right)$ and 9 point charges holding $Z=10$ are almost equal.

Fig. 5 shows the nematic order parameter in dependence on the added salt concentration for rods of length $L=10 D$, for different internal pitches and two different surface charges. With higher salt concentration, the orientational order decreases and the isotropic-nematic (IN) transition therefore shifts to higher rod concentration in accordance with the experimental results given in Ref. 43. The salt concentration changes the screening length of the Yukawa potential; a higher salt concentration leads to a shorter screening length, so the rods have to get closer (higher volume fraction) before they get aligned.

The limiting case of infinite salt concentration leads to zero screening length and thus reduces the system back to the hard spherocylinder case. For the particle volume fraction of 0.245 , this means that the isotropic phase is reached for high $c_{s}$ independent of the internal pitch and surface charge. (We chose to show the results for $\eta=0.245$, because different combinations of $Z^{s}, p^{\text {int }}$, and $c_{s}$ allow us to change the phase from nematic to isotropic at this density.) Increasing surface charge shifts the IN transition to lower rod concentration in contradiction to the experimental results; ${ }^{43}$ the reason for this is discussed in Ref. 43. It is based on a nonmonotonicity in the nematic order as a function of the total surface charge. The internal pitch dependence is the same as

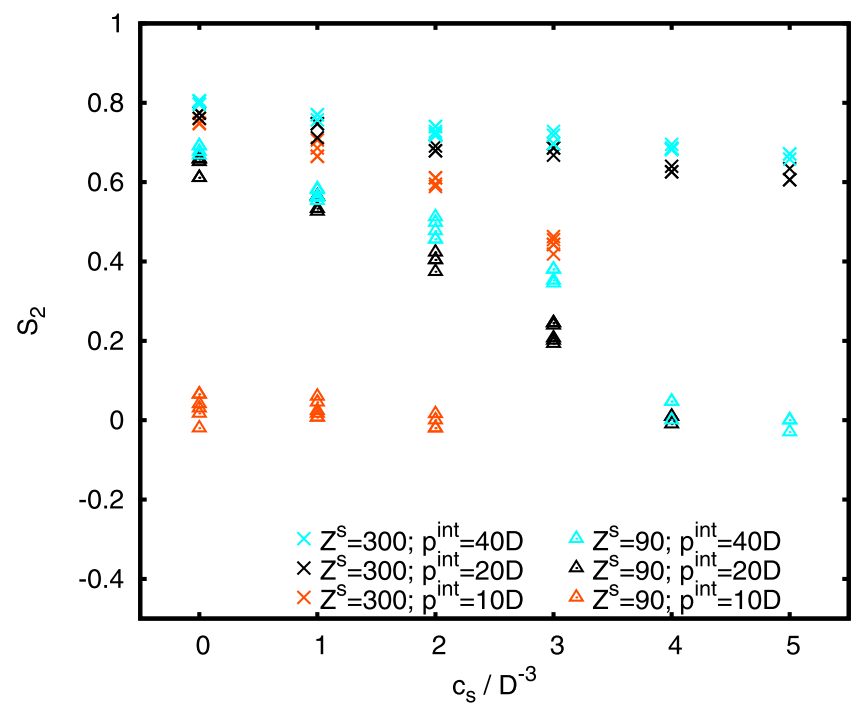

FIG. 5. Nematic order parameter vs. added salt concentration $\left(L=10 D, n^{\text {pc }}\right.$ $=9$, different internal pitches $p^{\text {int }}$, and two different surface charges $Z^{s}$ ) at a rod volume fraction of $24.5 \%$. 
discussed above-increased pitch leads to increased nematic order.

\section{B. Cholesteric pitches}

Next we apply the new method to compute equilibrium cholesteric pitches for different parameter values. The pitches are determined in two ways: On one hand, the MC move that changes the angle $\Psi$ relaxes the system into the equilibrium value $P=2 \pi L_{z} / \Psi_{\text {eq }}$, and on the other hand, a linear fit to the $z$-dependence of the angle of the nematic director with respect to an arbitrary direction, $\phi_{d}$, gives $P=2 \pi / a$, where $a$ is the slope of the fit. In Fig. 6 we plotted the values obtained by both methods for direct comparison. The errorbars for the pitch from cross boundary rotation are determined from the standard deviation of $\Psi$ during the production run. The errorbars for the pitch from linear fits to $\phi_{d}$ are determined from the uncertainties of the slope $a$ given by the fitting routine.

Fig. 6 shows the pitch as a function of rod concentration (for $L=20 D$ ). The pitch first fluctuates around $P=-200 D$,
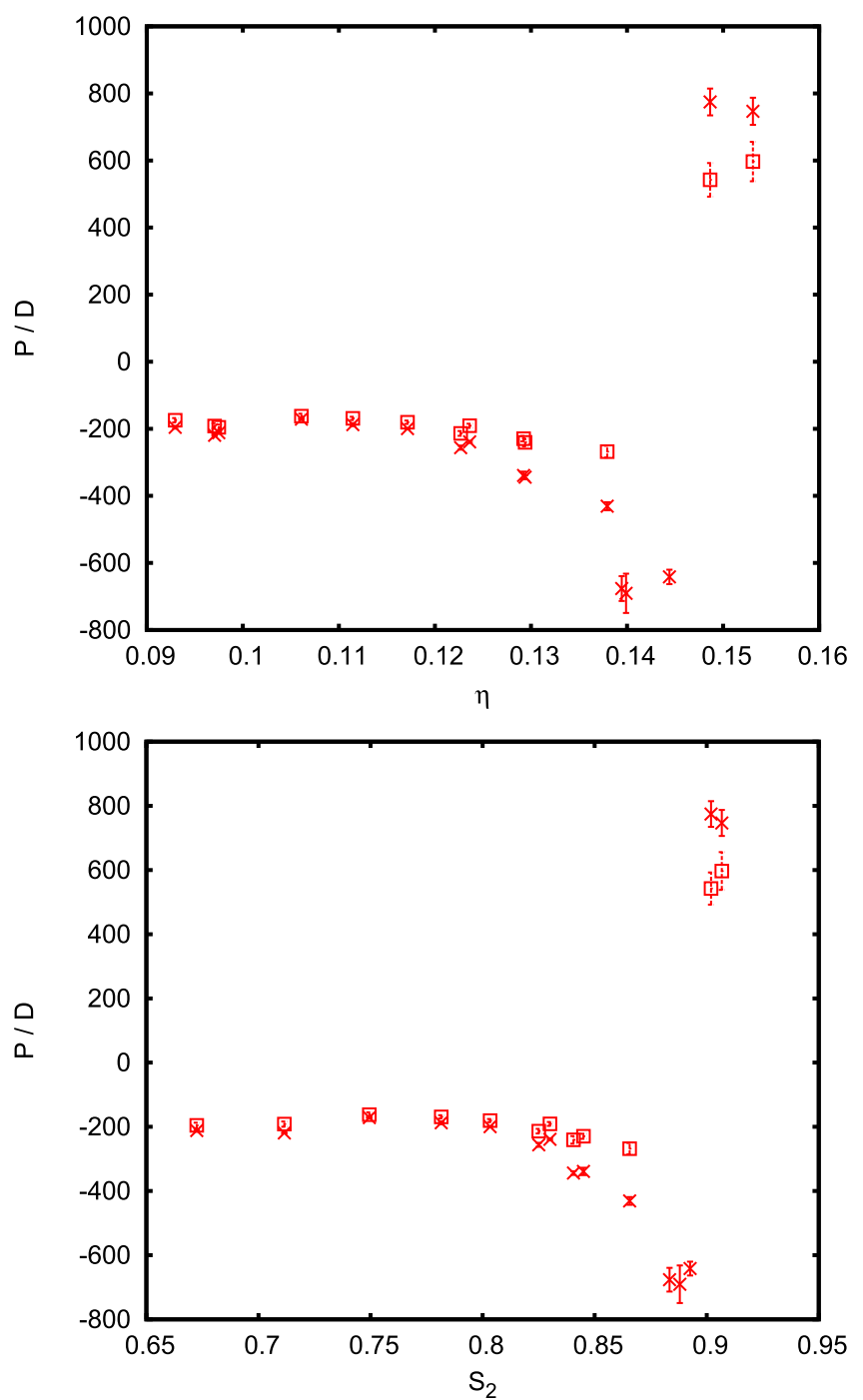

FIG. 6. Cholesteric pitch vs. volume fraction (top) and the nematic order parameter (bottom). Crosses show results from linear fits of $\phi_{d}$ and boxes from cross boundary rotation $\Psi_{\mathrm{eq}}$, as explained in the text. $\left(L=20 D, n^{\mathrm{pc}}=33\right.$, $\left.Z=10, c_{s}=1 D^{-3}, p^{\text {int }}=10 D\right)$. but then increases for higher concentrations and even shows sense inversion. Thus a particle of a given helical sense can produce cholesteric phases of both helical senses, depending on the concentration. In experiments, one mostly finds decreasing pitch in the low density regime. ${ }^{43}$ The higher density regime is often not accessible in experiments because of kinetic arrest. This problem does not occur in the simulations: First, our initial configurations are in the nematic phase, where the rods are already aligned and do not cage each other. Second, our systems are not large enough to build cholesteric domains with different orientations, which could prohibit reorientation on a large scale. But we need to remark that for volume fractions above $\eta=0.14$, boundary effects are present, so that the cholesteric pitch is not constant throughout the box, cf. the Appendix, which, however, does not alter the sense inversion behaviour.

Similar results were obtained for the systems of hard helices that showed that pitch inversion is largely driven by entropy. ${ }^{20-22}$ In addition, the sense inversion has been conjectured theoretically. ${ }^{44}$ Wensink showed that the angleaveraged chiral potential between two rods shows local
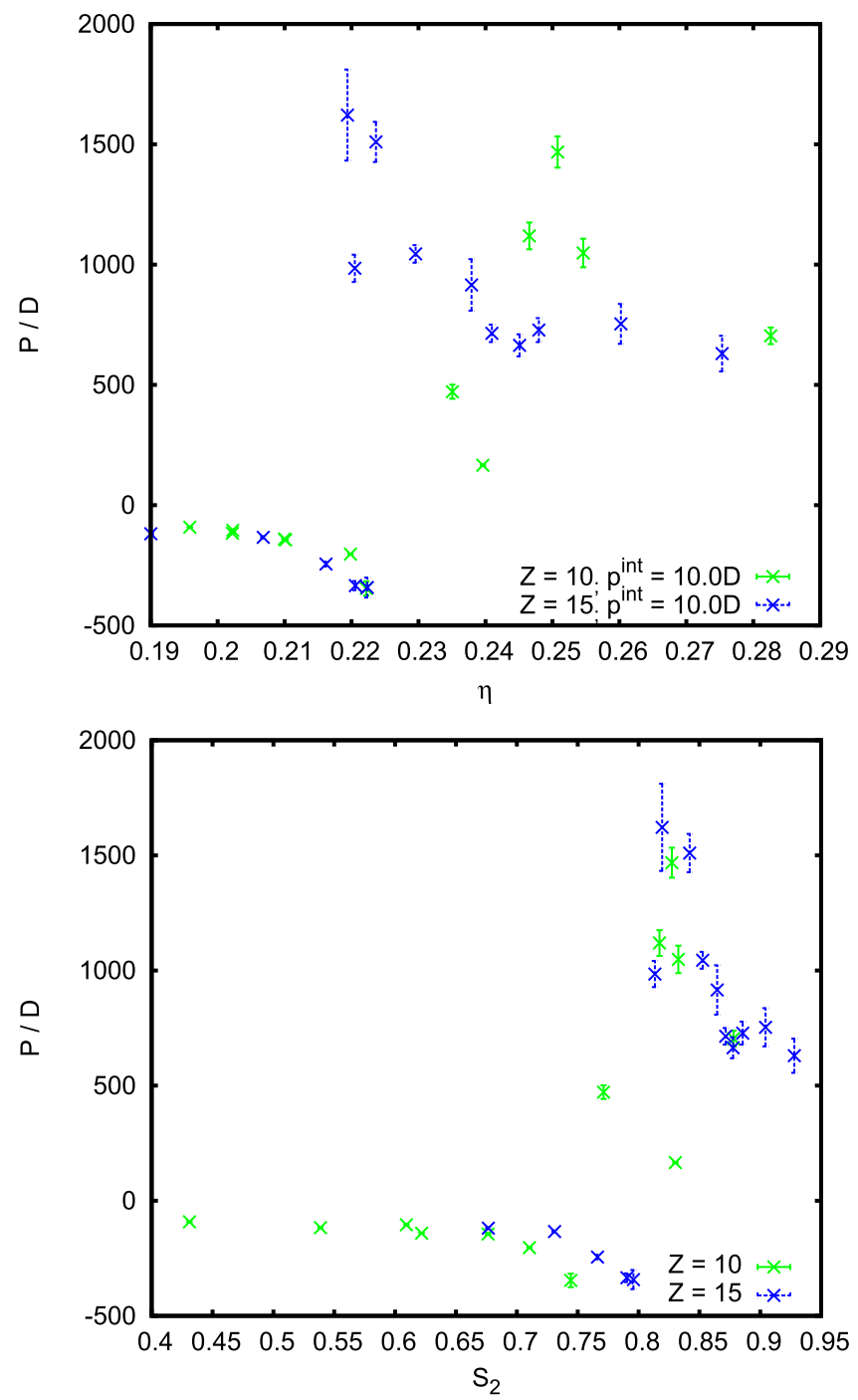

FIG. 7. Cholesteric pitch vs. volume fraction (top) and the nematic order parameter (bottom) $\left(L=10 D, n^{\mathrm{pc}}=17, Z\right.$ as indicated, $c_{s}=1 D^{-3}$, $\left.p^{\text {int }}=10 D\right)$. 

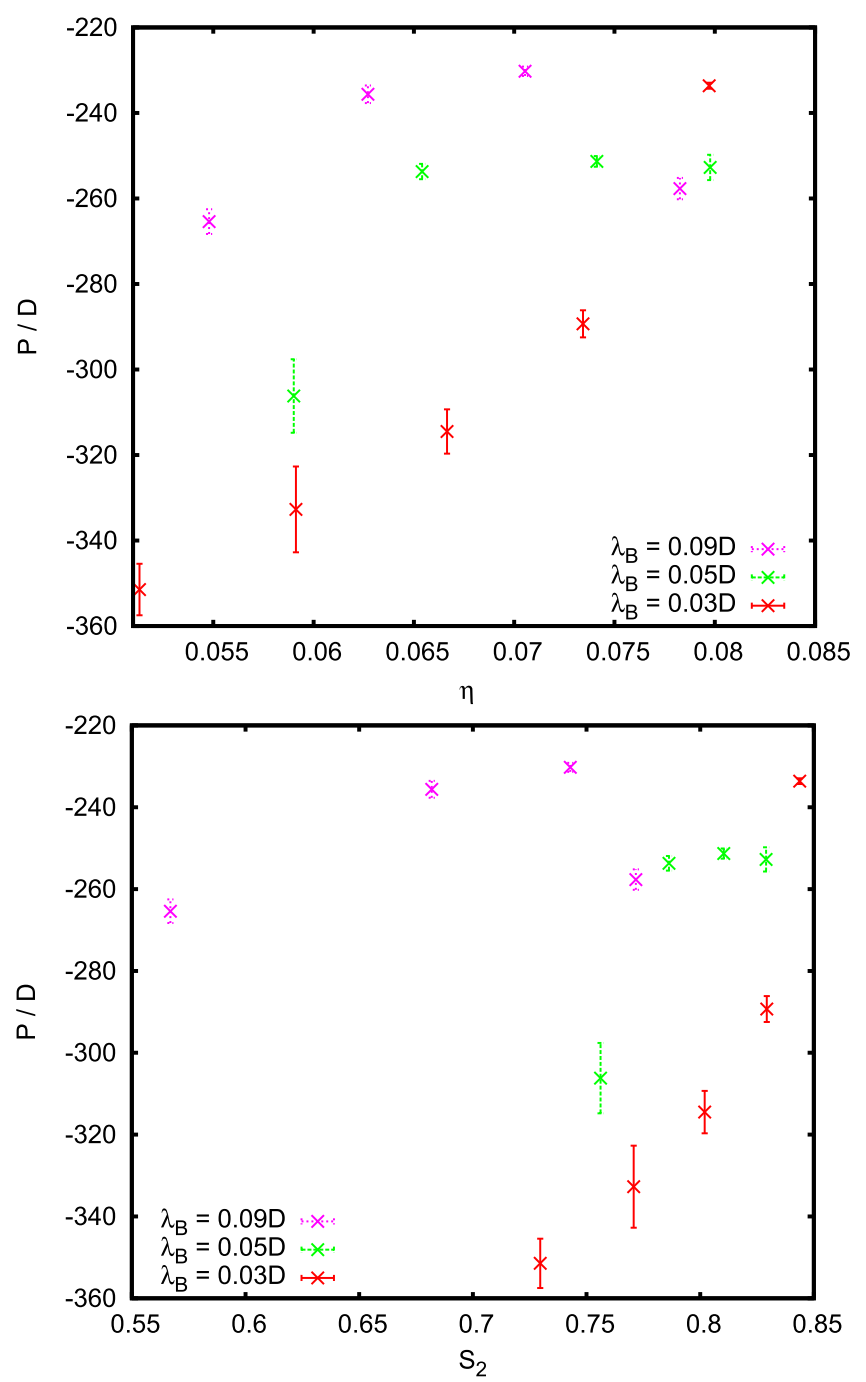

FIG. 8. Cholesteric pitch vs. volume fraction (top) and the nematic order parameter (bottom) $\left(N=1734, L=20 D, n^{\mathrm{pc}}=17, Z^{s}=690\right.$, different Bjerrum lengths $\lambda_{B}$ and internal pitch $p^{\text {int }}=20 D$ ).

minima at small twist angles, which for certain values of the internal pitch have the opposite sign of the angle at the global minimum. These minima in energy are only local, but can be stabilized entropically, as is shown by our simulation results as well as results of a recent, similar study in Ref. 29.

We also find the sense inversion for shorter rods with different charge strengths, as shown in Fig. 7, where the inversion happens at lower volume fraction for the larger charges. After the sense inversion, the cholesteric pitch decreases clearly with increasing volume fraction and order parameter.

With decreasing Bjerrum length, the cholesteric pitch at a low concentration increases, as shown in Fig. 8. For higher concentrations the pitch fluctuates around $P=-200 D$ independent of $\lambda_{B}$ (not shown). Since the Bjerrum length contributes to both, the amplitude of the interaction potential and the screening length, this monotonic behaviour is not expected to hold for all values of $\lambda_{B}$. An example for contradicting behaviour is found in the experimental results in Ref. 45, where a decreasing Bjerrum length models an increasing relative permittivity and that lowers the cholesteric pitch.

\section{CONCLUSION}

In this paper, we addressed two aspects of a system of helical Yukawa rods as a model for chiral liquid crystals: First, we presented the dependence of the location of the isotropic-nematic transition on the internal pitch and the concentration of added salt. Nematic order is enhanced as the pitch is increased or the salt concentration is decreased. In the second part, we introduced a new method to simulate cholesteric phases using soft walls and self-determined boundary conditions. Using this method, we found sense inversion of the cholesteric pitch, which confirms analytical predictions. $^{44}$

\section{ACKNOWLEDGMENTS}

We are grateful to Jan Lagerwall, Rik Wensink, and Michael Allen for helpful discussions. The simulations presented in this paper were carried out using the HPC facilities of the University of Luxembourg — see http://hpc.uni.lu. ${ }^{46}$ Financial support is kindly acknowledged from the Luxembourg National Research Fund (FNR; project MISONANCE, Grant No. C14/MS/8331546).

\section{APPENDIX: ERROR ESTIMATION}

\section{Isotropic-nematic transition}

To check for finite size effects, the system size was doubled from $N=1800$ to $N=3600$. The result is shown in Fig. 9 . There is only one point for which the nematic order parameter differs significantly ( $p^{\text {int }}=40 D$ at the transition). Thus we use the smaller system size but keep in mind that there might be small changes for larger systems that should be studied in detail in the future.

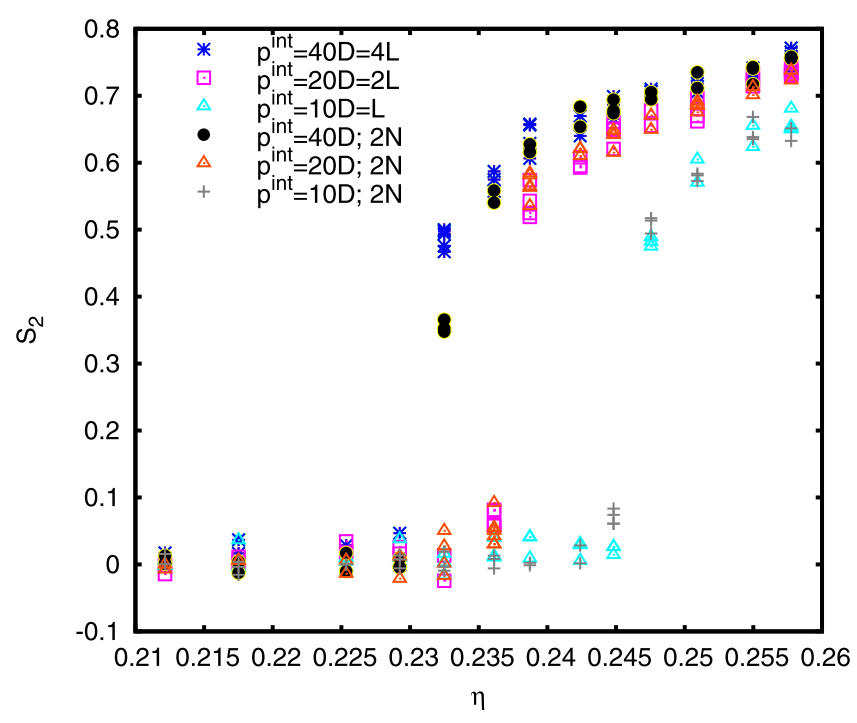

FIG. 9. Nematic order parameter vs. volume fraction for varying internal pitches $p^{\text {int }}$ and for two different system sizes, $N=1800$ and $3600(L=10 D$, $\left.n^{\mathrm{pc}}=9, Z=10, c_{s}=1 D^{-3}\right)$. 


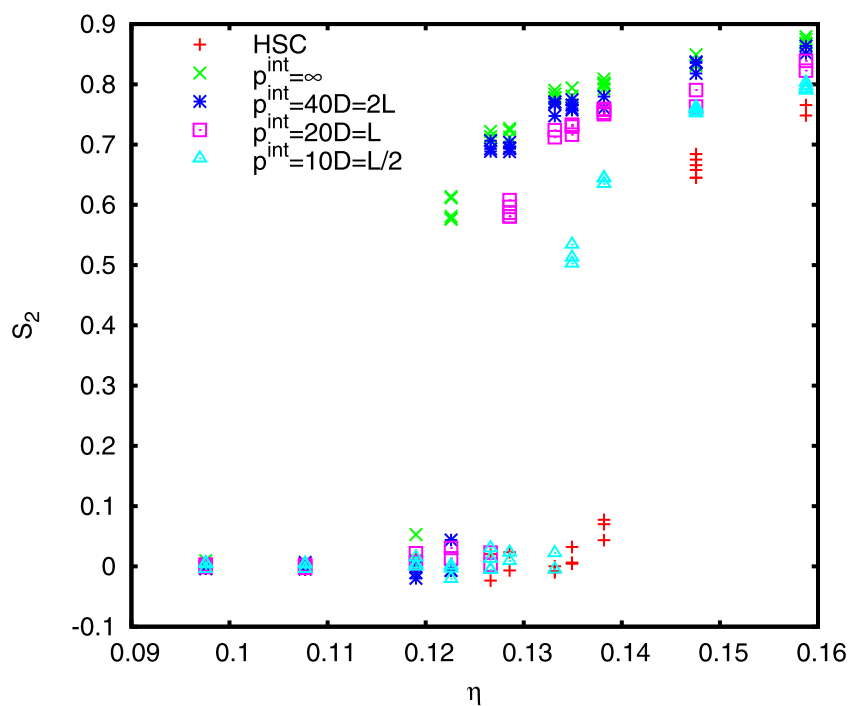

FIG. 10. Nematic order parameter vs. for varying internal pitches $p^{\text {int }}$ $\left(N=1800, L=20 D, n^{p c}=17, Z=10, c_{s}=1 D^{-3}\right)$.

The same pitch dependence as in Fig. 9 is seen for longer rods of length $L=20 D$, cf. Fig. 10 .

Especially for long internal pitches, the charges are concentrated on one side of the rod, which is not a realistic model for CNC. Therefore we investigated the behaviour of a double charge helix compared to the single helix that we use for most simulations. As can be seen in Fig. 11, the nematic order is increased, when two helices are present, but the IN transition itself is not changed. The surface charge and volume fraction range are taken from experimental results in Ref. 47, and with both, single and double helix, the IN transition is at the same volume fraction as found there. We conclude that it is sufficient to work with a single helix, since the calculations are much faster and there are only small deviations from the behaviour of a double helix.

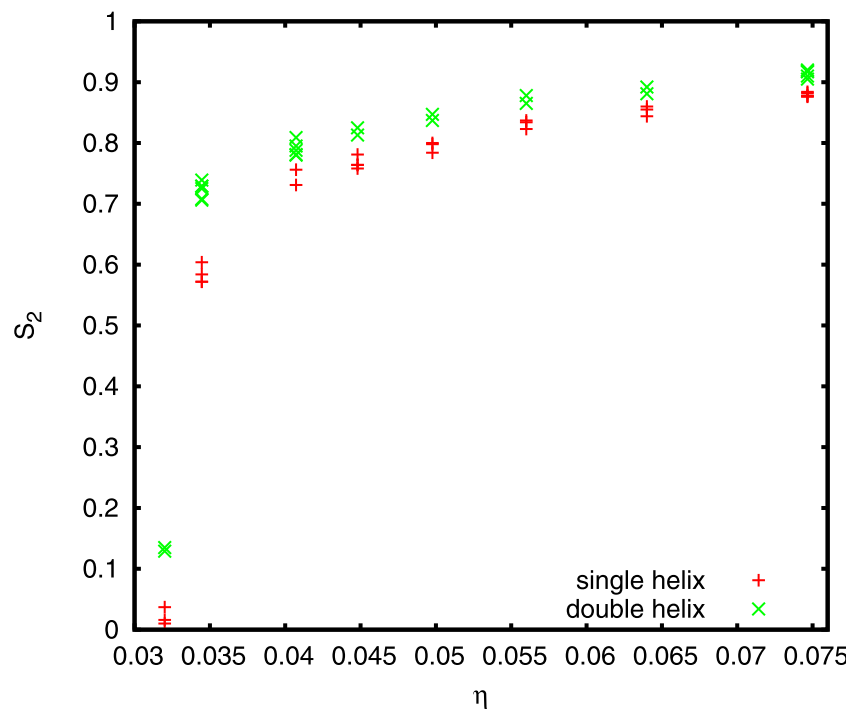

FIG. 11. Nematic order parameter vs. volume fraction for single and double charge helix $\left(N=1800, L=25 D, n^{\mathrm{pc}}=21, Z^{s}=750, c_{s}=0 D^{-3}, p^{\text {int }}\right.$ $=40 D$ ).

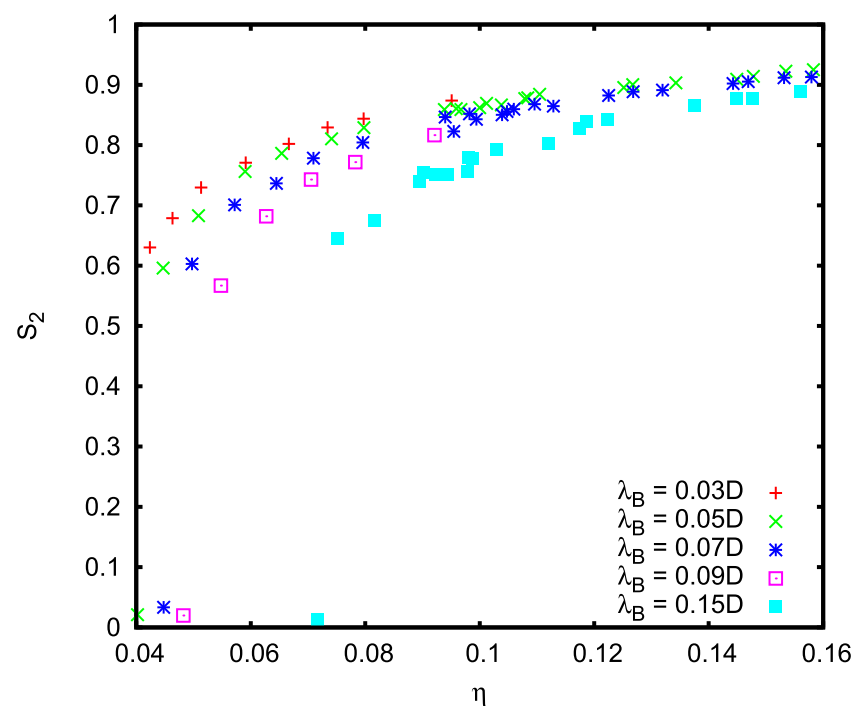

FIG. 12. Nematic order parameter vs. volume fraction for varying Bjerrum lengths $\lambda_{B}\left(L=20 D, n^{\mathrm{pc}}=17, Z^{s}=690, p^{\mathrm{int}}=20 D, c_{s}=0 D^{-3}\right)$.

\section{Boundary effects}

The most obvious effect of the new method is to change the volume accessible to the ends of the rods. The volume fraction of rods can now refer either to the hard volume for the centers of mass of the rods or to the soft volume for the ends of the rods; the latter is calculated as $V_{s}=V+L f L_{x} L_{y}$, where $V=L_{x} L_{y} L_{z}$ is the hard volume. We use the soft volume for the graphs shown here; using the hard volume would slightly shift the transition (in a non-linear way) to higher volume fractions. The small additional volume does not lead to qualitative changes in the behaviour of the system. As one example, Fig. 12 shows the isotropic-cholesteric (IC) transition for different Bjerrum lengths. With decreasing Bjerrum length, the nematic order increases. This result is similar to the experimental results in Ref. 45, where we already compared

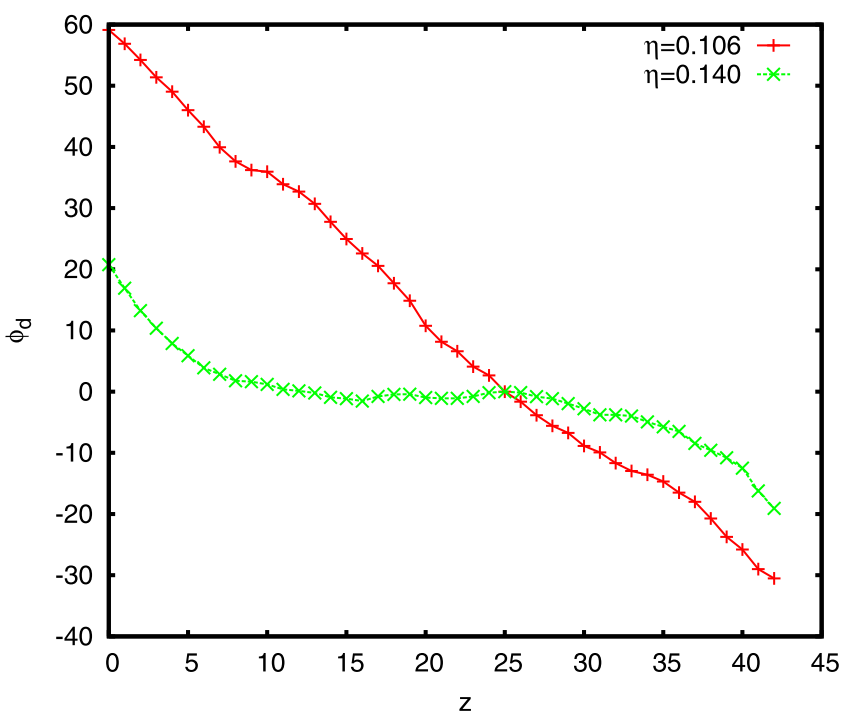

FIG. 13. Angle of the nematic director vs. position on the chiral axis for two representative examples of Fig. 6. The lower volume fraction example shows a perfect linear director rotation, while for the higher volume fraction, clear boundary effects are found, where the cholesteric pitch is smaller close to the walls. 
simulation and experimental results, but used the common periodic boundaries.

Although the walls are soft and allow for the rotation of rods, their influence is not completely removed. At high densities or strong interactions, we still find layering close to the walls, leading to different pitches at the center of the box than in the vicinity of the walls. This is an obvious effect and we can assign the relevant regions in the phase diagram. In most other cases, we find constant rotation of the nematic director throughout the box, see Fig. 13.

To estimate the errors produced by the unphysical interaction across the boundary (which is not invariant under the exchange of particle and periodic image, as explained in the main text), we compute the free energy difference between the center of the box and the vicinity of the walls. We attempt to swap a particle from the center with another particle somewhere in the box. Swapping includes exchanging the particles' positions and rotating their orientations according to their distance along the cholesteric axis and the equilibrium pitch value $\Psi_{\text {eq }}$ found in the simulation. We observe that the acceptance rate of the swap moves is higher for swapping a center and a wall particle than for swapping two center particles. The resulting free energy difference per particle (logarithm of the ratio of acceptance rates) is $\Delta F \approx 0.5 k_{B} T$. This quantity decreases with the distance from the wall, and for a distance of $2 D$, it is already reduced to $\Delta F \approx 0.02 k_{B} T$. Thus the range of boundary effects in the smallest box used for Fig. 6 is $9.3 \%$ of the whole box length.

${ }^{1}$ P. G. de Gennes and J. Prost, The Physics of Liquid Crystals, 2nd ed. (Clarendon Press, Oxford, 1993).

${ }^{2}$ R. Dreher and G. Meier, "Optical properties of cholesteric liquid crystals," Phys. Rev. A 8, 1616 (1973).

${ }^{3}$ P. Yeh and C. Gu, Optics of Liquid Crystal Displays (John Wiley \& Sons, 2010).

${ }^{4}$ A. Mujahid, H. Stathopulos, P. A. Lieberzeit, and F. L. Dickert, "Solvent vapour detection with cholesteric liquid crystals-Optical and mass-sensitive evaluation of the sensor mechanism," Sensors 10, 4887 (2010).

${ }^{5}$ A. Saha, Y. Tanaka, Y. Han, C. M. W. Bastiaansen, D. J. Broer, and R. P. Sijbesma, "Irreversible visual sensing of humidity using a cholesteric liquid crystal," Chem. Commun. 48, 4579 (2012).

${ }^{6}$ O. T. Picot, M. Dai, E. Billoti, D. J. Broer, T. Peijs, and C. W. M. Bastiaansen, "A real time optical strain sensor based on a cholesteric liquid crystal network," RSC Adv. 3, 18794 (2013).

${ }^{7}$ G. Solladié, P. Hugelé, R. Bartsch, and A. Skoulios, "Bildung von enantiomerenreinen flüssigkristallen aus axial-chiralen biphenylen," Angew. Chem. 108, 1640 (1996).

${ }^{8}$ H. Kosho, S. Hiramatsu, T. Nishi, Y. Tanaka, S. Kawauchi, and J. Wanabe, "Thermotropic cholesteric liquid crystals in ester derivatives of hydroxypropylcellulose," High Perform. Polym. 11, 41 (1999).

${ }^{9}$ W. R. Krigbaum, A. Ciferri, J. Asrar, H. Toriumi, and J. Preston, "A polyester forming a thermotropic cholesteric phase," Mol. Cryst. Liq. Cryst. 76, 79 (1981).

${ }^{10} \mathrm{~J}$. Watanabe, M. Goto, and T. Nagase, "Thermotropic polypeptides. 3. Investigation of cholesteric mesophase properties of poly $(\gamma$-benzyl Lglutamate-co- $\gamma$-dodecyl L-glutamates) by circular dichroic measurements," Macromolecules 20, 298 (1987).

${ }^{11} \mathrm{~F}$. Livolant, "Ordered phases of DNA in vivo and in vitro," Physica A 176, 117 (1991)

${ }^{12} \mathrm{E}$. Grelet and S. Fraden, "What is the origin of chirality in the cholesteric phase of virus suspensions?," Phys. Rev. Lett. 90, 198302 (2003).

${ }^{13}$ Z. Dogic and S. Fraden, "Ordered phases of filamentous viruses," Curr. Opin. Colloid Interface Sci. 11, 47 (2006).

${ }^{14}$ J. P. F. Lagerwall, C. Schütz, M. Salajkova, J. Noh, J. H. Park, G. Scalia, and L. Bergström, "Cellulose nanocrystal-based materials: From liquid crystal self-assembly and glass formation to multifunctional thin films," NPG Asia Mater. 6, e80 (2014).

${ }^{15}$ L. Onsager, "The effects of shape on the interaction of colloidal particles," Ann. N. Y. Acad. Sci. 51, 627 (1949).

${ }^{16}$ J. P. Straley, "Theory of piezoelectricity in nematic liquid crystals, and of the cholesteric ordering," Phys. Rev. A 14, 1835 (1976).

${ }^{17}$ H. H. Wensink and G. Jackson, "Cholesteric order in systems of helical Yukawa rods," J. Phys.: Condens. Matter 23, 194107 (2011).

${ }^{18}$ J. D. Parsons, "Nematic ordering in a system of rods," Phys. Rev. A 19, 1225 (1979)

${ }^{19}$ S.-D. Lee, "A numerical investigation of nematic ordering based on a simple hard-rod model," J. Chem. Phys. 87, 4972 (1987).

${ }^{20}$ E. Frezza, A. Ferrarini, H. B. Kolli, A. Giacometti, and G. Cinacchi, "Left or right cholesterics? A matter of helix handedness and curliness," Phys. Chem. Chem. Phys. 16, 16225 (2014).

${ }^{21}$ S. Belli, S. Dussi, M. Dijkstra, and R. van Roij, "Density functional theory for chiral nematic liquid crystals," Phys. Rev. E 90, 020503 (2014).

${ }^{22}$ S. Dussi, S. Belli, R. van Roij, and M. Dijkstra, "Cholesterics of colloidal helices: Predicting the macroscopic pitch from the particle shape and thermodynamic state," J. Chem. Phys. 142, 074905 (2015).

${ }^{23}$ M. P. Allen, "Density functional theory for chiral nematic liquid crystals," Mol. Phys. 114, 2574 (2016).

${ }^{24} \mathrm{R}$. Memmer, "Determination of equilibrium pitch of cholesteric phases by isobaric-isothermal Monte Carlo simulation," J. Chem. Phys. 114, 8210(2001).

${ }^{25}$ G. Germano, M. P. Allen, and A. J. Masters, "Simultaneous calculation of the helical pitch and the twist elastic constant in chiral liquid crystals from intermolecular torques," J. Chem. Phys. 116, 9422 (2002).

${ }^{26} \mathrm{~S}$. Varga and G. Jackson, "Simulation of the macroscopic pitch of a chiral nematic phase of a model chiral mesogen," Chem. Phys. Lett. 377, 6 (2003).

${ }^{27}$ E. Frezza, A. Ferrarini, H. B. Kolli, A. Giacometti, and G. Cinacchi, "The isotropic-to-nematic phase transition in hard helices: Theory and simulation," J. Chem. Phys. 138, 164906 (2013).

${ }^{28}$ H. B. Kolli, E. Frezza, G. Cinacchi, A. Ferrarini, A. Giacometti, and T. S. Hudson, "Communication: From rods to helices: Evidence of a screw-like nematic phase," J. Chem. Phys. 140, 081101 (2014).

${ }^{29}$ S. Rủžička and H. H. Wensink, "Simulating the pitch sensitivity of twisted nematics of patchy rods," Soft Matter 12, 5205 (2016).

${ }^{30}$ S. Dussi and M. Dijkstra, "Entropy-driven formation of chiral nematic phases by computer simulations," Nat. Commun. 7, 11175 (2016).

${ }^{31}$ J. F. Revol and R. H. Marchessault, "In vitro nematic ordering of chitin crystallites,” Int. J. Biol. Macromol. 15, 329 (1993).

${ }^{32}$ S. B. Murray and A. C. Neville, "The role of $\mathrm{pH}$, temperature and nucleation in the formation of cholesteric liquid crystal spherulites from chitin and chitosan,” Int. J. Biol. Macromol. 22, 137 (1998).

${ }^{33}$ L. Rossi, S. Sacanna, and K. P. Velikov, "Cholesteric colloidal liquid crystals from phytosterol rod-like particles," Soft Matter 7, 64 (2011).

${ }^{34}$ A. Poniewierski and R. Hołyst, "Nematic alignment at a solid substrate: The model of hard spherocylinders near a hard wall," Phys. Rev. A 38, 3721 (1988).

${ }^{35}$ A. Poniewierski, "Ordering of hard needles at a hard wall," Phys. Rev. E 47, 3396 (1993).

${ }^{36}$ M. Dijkstra, R. van Roij, and R. Evans, "Wetting and capillary nematization of a hard-rod fluid: A simulation study,” Phys. Rev. E 63, 051703 (2001).

${ }^{37}$ R. van Roij, M. Dijkstra, and R. Evans, "Interfaces, wetting, and capillary nematization of a hard-rod fluid: Theory for the Zwanzig model," J. Chem. Phys. 113, 7689 (2000).

${ }^{38} \mathrm{~J}$. Vieillard-Baron, "The equation of state of a system of hard spherocylinders," Mol. Phys. 28, 809 (1974).

${ }^{39}$ M. P. Allen and A. J. Masters, "Computer simulation of a twisted nematic liquid crystal," Mol. Phys. 79, 277 (1993).

${ }^{40}$ J. S. Lintuvuori, "Towards improved simulations of self-organising molecular materials," Durham thesis, Durham University, 2009, available at Durham E-Thesis Online: http://etheses.dur.ac.uk/56/.

${ }^{41}$ W. M. Saslow, M. Gabay, and W.-M. Zhang, "Spiraling algorithm: Collective Monte Carlo trial and self-determined boundary conditions for incommensurate spin systems," Phys. Rev. Lett. 68, 3627 (1992).

${ }^{42}$ R. Memmer and F. Janssen, "Computer simulation of chiral liquid crystal phases. Part 5: Temperature dependence of the pitch of a cholesteric phase studied under self-determined boundary conditions,"J. Chem. Soc., Faraday Trans. 94, 267 (1998); "Computer simulation of chiral liquid crystal phases 
VI. A temperature induced helix inversion in a single component cholesteric liquid crystal," Liq. Cryst. 24, 805 (1998).

${ }^{43}$ C. Honorato-Rios, A. Kuhnhold, J. R. Bruckner, R. Dannert, T. Schilling, and J. P. F. Lagerwall, "Equilibrium liquid crystal phase diagrams and detection of kinetic arrest in cellulose nanocrystal suspensions," Front. Mater. 3, 21 (2016).

${ }^{44}$ H. H. Wensink, "Spontaneous sense inversion in helical mesophases," EPL 107, 36001 (2014).

${ }^{45}$ J. R. Bruckner, A. Kuhnhold, C. Honorato-Rios, T. Schilling, and J. P. F. Lagerwall, "Enhancing self-assembly in cellulose nanocrystal suspensions using high-permittivity solvents," Langmuir 32, 9854 (2016). ${ }^{46} \mathrm{~S}$. Varrette, P. Bouvry, H. Cartiaux, F. Georgatos, "Management of an academic HPC cluster: The UL experience," in Proceedings of the 2014
International Conference on High Performance Computing \& Simulation (HPCS 2014) (IEEE, 2014), p. 959.

${ }^{47}$ S. Beck-Candanedo, M. Roman, and D. R. Gray, "Effect of reaction conditions on the properties and behavior of wood cellulose nanocrystal suspensions," Biomacromolecules 6, 1048 (2005).

${ }^{48}$ Note: If $n$ is small while $S_{w}-S_{a v}$ is large, the walls might get too soft, resulting in a lower orientational order at the walls compared to the average, which is problematic since the rods need to be pushed back again by harder (less soft) walls. Therefore $n$ needs to be large enough to increase the walls' softness only slowly.

${ }^{49} \mathrm{~A}$ similar approach to reduce the influence of a hard wall on the behaviour of rods has been used in a different context in Ref. 36, where wetting of hard spherocylinders on a single hard wall has been studied. 